\title{
Low-Cost and Programmable CRC Implementation based on FPGA (Extended Version)
}

\author{
Huan Liu, Zhiliang Qiu, Weitao Pan, Jun Li, Ling Zheng and Ya Gao
}

\begin{abstract}
Cyclic redundancy check (CRC) is a well-known error detection code that is widely used in Ethernet, PCIe, and other transmission protocols. The existing FPGA-based implementation solutions are faced with the problem of excessive resource utilization in high-performance scenarios. The padding zeros problem and the introduction of programmability further exacerbate this problem. In this brief, the stride-by-5 algorithm is proposed to achieve the optimal utilization of FPGA resources. The pipelining go back algorithm is proposed to solve the padding zeros problem. The method of reprogramming by HWICAP is proposed to realize programmability with a small and constant resource utilization. The experimental results show that the resource utilization of proposed non-segmented architecture is 80.7\%-87.5\% and $25.1 \%-46.2 \%$ lower than those of two stateof-the-art FPGA-based CRC implementations, and the proposed segmented architecture has a lower resource utilization by $81.7 \%$ 85.9\% and $2.9 \%-20.8 \%$ compared wtih the two state-of-the-art architectures; meanwhile, the throughput and programmability are guaranteed. We made the source code available on GitHub[1].
\end{abstract}

Index Terms-Cyclic redundancy check, FPGA, low cost, programmable, HWICAP.

\section{INTRODUCTION}

As the throughput of networks is on a constant rise, increasingly more packet processing tasks are being offloaded to the FPGA-based SmartNIC[2], including the generation and verification of cyclic redundancy check (CRC). The 400G and the coming multi-terabit Ethernet demand faster CRC caculations, and the implementation of high-performance CRC calculations based on FPGAs needs to meet three requirements: 1) Reduce parallelization cost. The end of Dennard scaling[3] results in a bottleneck for improving the frequency of integrated circuits, and higher throughput means a wider bus inside chips. The slicing-by-4 and slicing-by- 8 algorithms are proposed for parallel processing in[4], which is suitable for CPU but not optimal for FPGA[5]. 2) Solve the padding zeros problem. The parallelization means that the final word of a transaction is composed of valid bytes along with padding

This work is supported in part by the National Key Laboratory Foundation (HTKJ2019KL504012), National Natural Science Foundation (61502204).

H. Liu, Z. Qiu, and W. Pan are with the State Key Laboratory of Integrated Service Networks, Xidian University, Xi'an 710071, China (e-mail: huanliu@stu.xidian.edu.cn).

J. Li is with the National Key Laboratory of Science and Technology on Space Microwave, China Academy of Space Technology (Xi'an), Xi'an 710100, China.

Ling Zheng is with the School of Communication and Information Engineering, Xi'an University of Posts and Telecommunications, Xi'an 710121, China.

Ya Gao is with the School of Internet of Things Technology, Wuxi Institute of Technology, Wuxi 214121, China.

Corresponding author: Weitao Pan (wtpan@mail.xidian.edu.cn) zeros. The number of padding zeros is uncertain, and CRC calculations using the complete final word would cause an erroneous result, which is called the padding zeros problem. Multiple tables can be used to process the final word, and every table corresponds to a possible length of valid bytes[6]. The scheme introduces an $O\left(n^{2}\right)$ resource utilization when the bus width is $n$ bits. [7][8] is one of the state-of-the-art schemes for solving this problem. The tables for the final word are organized in the manner of a pipeline, and each pipeline step corresponds to one layer of a binary search tree. An $O(n)$ resource utilization is introduced. 3) Keеp programmability. A programmable implementation of the CRC algorithm can achieve better reusability; thus, a wide range of applications can be supported without circuit modification. The demand can be found in iSCSI[9] and P4[10]. A specific circuit architecture is used to guarantee programmability[11][12], but it is not suitable for FPGA. [5] is one of the state-of-the-art schemes that is suitable for FPGA, but it requires a complex configuration circuit that leads to a large resource utilization increase with the bus width.

All three of the aforementioned requirements lead to a considerable resource utilization. Although slicing[4][5], aggressive strides, simultaneous processing of multiple streams[7] and many other principles behind CRC acceleration are well known, they can't achieve low cost, high performance and programmability at the same time. A multi-core, multi-socket system with Intel's CRC instruction[13] can achieve high throughput, but they suffer from high latency and high power consumption in packet processing applications. In this brief, two algorithms and a method corresponding to the three requirements are proposed to decrease the resource utilization with guaranteed throughput and programmability. First, the stride-by-5 algorithm is proposed, which can reduce the resource utilization by $79.69 \%-79.98 \%$ compared with the slicing-by-4 and slicing-by-8 algorithms. Second, the pipelining go back algorithm is proposed to solve the padding zeros problem, which will introduce an $O\left(\log _{2} n\right)$ resource utilization. Finally, a hardware internal configuration access port (HWICAP) is used to realize dynamic programmability, and it leads to a small and constant resource utilization regardless of the bus width.

The remainder of this brief is organized as follows. Section II provides preliminaries to our proposals. Section III discusses the system architecture and the three proposals. Section IV shows the synthesis results and the board-level implementation results. Section V concludes this brief. 


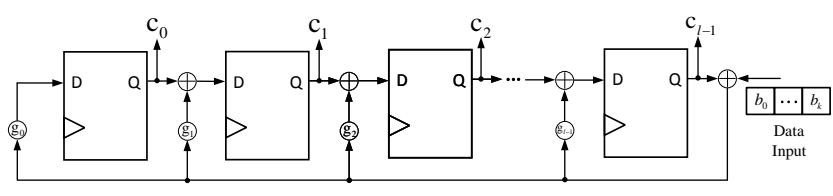

Fig. 1: LFSR for CRC computing.

\section{PRELIMINARIES}

\section{A. FPGA LUT Architecture}

The basic logic resource of modern Xilinx FPGAs is lookup tables (LUTs), which can be considered a RAM with five inputs and two outputs[14]. A truth table can be stored in a LUT, and two logical equations with the same five inputs can be realized using it. This is an important property that will be used in the stride-by-5 algorithm. LUT is the most consumed resource in FPGA-based CRC implementations, and the number of consumed LUTs is used as the indicator of the resource utilization.

\section{B. Serial and Parallel CRC Algorithms}

\section{Serial CRC Algorithm}

The CRC algorithm is a long division performed with modulo- 2 arithmetic. The dividend is a polynomial $B(x)$ whose coefficient is the input data. The divisor is a given polynomial $G(x)$, and the coefficient of the remainder $R(x)$ is the wanted CRC value. Addition and subtraction can be realized by the xor operation in $\mathrm{GF}(2)$, and "+" means xor in the remainder of this brief. The aforementioned division can be realized by the linear feedback shift register (LFSR), as shown in Fig. 1.

The coefficient of $G(x)$ is $\left[g_{l}, g_{l-1}, \ldots, g_{0}\right]$. The coefficient of $B(x)$ is $\left[b_{0}, b_{1}, \ldots, b_{k}\right]$, with $b_{0}$ being the most significant bit. The initial value of the LFSR is $C^{(0)}=$ $\left[\mathbf{c}_{l-1}^{(0)}, \mathbf{c}_{l-2}^{(0)}, \cdots, \mathbf{c}_{0}^{(0)}\right]^{T}$. The value of the LFSR is $C^{(m)}$ when bit $b_{m-1}$ enters the LFSR, and it will become $C^{(m+1)}$ after bit $b_{m}$ enters the LFSR. We can obtain the relationship between $C^{(m)}$ and $C^{(m+1)}$ from Fig. 1, which is

$$
C^{(m+1)}=T C^{(m)}+S b_{m}
$$

where $T$ is a matrix of size $l \times l . S$ is a column vector of size $l$, and

$$
\begin{gathered}
T=\left[\begin{array}{ccccc}
g_{l-1} & 1 & 0 & \ldots & 0 \\
g_{l-2} & 0 & 1 & \ldots & 0 \\
\cdots & \ldots & \ldots & \cdots & \ldots \\
g_{1} & 0 & 0 & \cdots & 1 \\
g_{0} & 0 & 0 & \cdots & 0
\end{array}\right] \\
S=\left[\mathrm{g}_{l-1}, \mathrm{~g}_{l-2}, \ldots, \mathrm{g}_{0}\right]^{T}
\end{gathered}
$$

Parallel CRC Algorithm

The parallel CRC algorithm can process multiple data input bits simultaneously[15], and its theoretical equation can be derived from (1). The number of bits processed in parallel is $n$, which is also the width of the inner bus in the remainder of this brief. The parallel input data are $B_{n}=\left[b_{0}, b_{1}, \ldots, b_{n-1}\right]^{T}$.
The value of the LFSR is $C^{(k)}$ before $B_{n}$ enters. The relationship between $C^{(n+k)}$ and $C^{(k)}$ is

$$
\begin{aligned}
C^{(n+k)} & =T C^{(n+k-1)}+S b_{n-1} \\
& =T^{n} C^{(k)}+T^{n-1} S b_{0}+T^{n-2} S b_{1}+\cdots+S b_{n-1} \\
& =T^{n} C^{(k)}+W_{l n} B_{n}
\end{aligned}
$$

where $W_{l n}$ is a matrix of size $l \times n$ and

$$
W_{l n}=\left[T^{n-1} S, T^{n-2} S, \ldots, T S, S\right]
$$

$T^{n}$ and $W_{l n}$ can be calculated by equations (2), (3) and (5) after $G(x)$ is given, and parallel processing by $n$ bits can be achieved by equation (4).

\section{Programmability and HWICAP}

$T^{n}$ and $W_{l n}$ are generally stored inside LUTs for the FPGA-based implementation of CRC algorithms, and a programmable implementation requires the ability to modify the content of the LUTs at runtime. Previous research using logic resources (LUTs and registers) to realize configuration logic would lead to several thousands of LUTs consumed when $n \geq 1024$ [5].

HWICAP is an Xilinx IP core that can afford users with access to ICAP primitives using the AXI4-Lite protocol[14]. It can modify the content of the LUTs dynamically. The resource utilization of HWICAP is as low as 186 LUTs, and it will not increase with increasing inner bus width. For the Intel/Altera FPGAs, similar function can be achieved by using PR-IP[16][17].

\section{Proposed WorK}

\section{A. Non-Segmented System Architecture}

The proposed non-segmented system architecture is shown in Fig. 2. Non-segmented system architecture means that there should be one frame in a single word, and segmented system architecture can process multiple frames at the same time[18]. Region 1 and Region 2 correspond to the computation of $W_{l n} B_{n}$ in (4). Region 1 consumes most of the LUTs, and the number of consumed LUTs linearly depends on the size of $W_{l n}$. The stride-by-5 algorithm, which is discussed in Section $\mathrm{B}$, is proposed to reduce the LUT consumption of Region 1. Region 2 is implemented by the means of an xor tree instead of a one-stage xor function to achieve higher performance. Region 3 completes the computation of (4). It consumes few LUTs for the small size of $\boldsymbol{T}^{n}$. The padding zeros problem is solved by Region 4, and the pipelining go back algorithm, which results in an $O\left(\log _{2} n\right)$ resource utilization, is proposed and discussed in Section C. Region 5 is a HWICAP controller that can modify the content of the LUTs dynamically. The operation procedure is discussed in Section D. A segmented system architecture is proposed in Section E. The packet processing flow of the two system architectures are illustrated in Section F. 


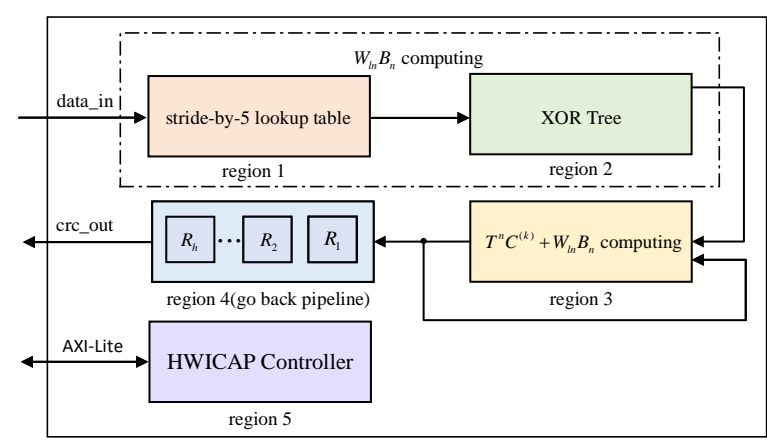

Fig. 2: Proposed non-segmented system architecture.

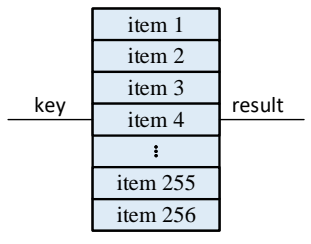

(a)

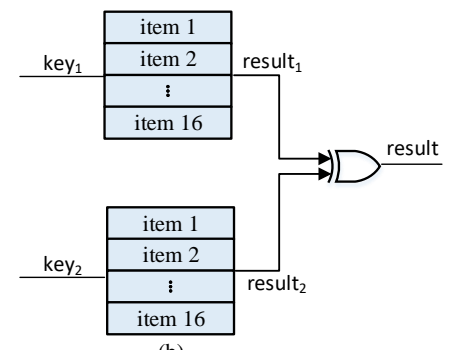

(b)
Fig. 3: Function implementation with (a) stride-by-8 and (b) stride-by-4.

\section{B. Stride-by-5 Algorithm}

In this section, the model of the resource utilization is established, the stride-by-5 is proven to be the best stride for various bus widths, and the stride-by- 5 algorithm is described in Algorithm 1.

Stride, as its name implies, means the number of bits processed by a single logical table[19]. The logical table can be realized using FPGA LUTs, and it can load the truth table of a function. For example, an 8-input function is defined as

$$
y=x_{1}+x_{2}+x_{3}+x_{4}+x_{5}+x_{6}+x_{7}+x_{8}
$$

which can be transformed equivalently as

$$
\left\{\begin{array}{l}
y_{1}=x_{1}+x_{2}+x_{3}+x_{4} \\
y_{2}=x_{5}+x_{6}+x_{7}+x_{8} \\
y=y_{1}+y_{2}
\end{array}\right.
$$

Equations (6) and (7), whose strides are 8 and 4, can be implemented as shown in Fig. 3(a) and Fig. 3(b), respectively. A smaller stride means that a smaller logical table can be realized by a single LUT or cascaded LUTs. Can stride-by1 be considered the best stride for FPGA implementation? We will establish the resource utilization model and find the answer.

$l$ equations with the same $n$ inputs are required to realize the computation of $W_{l n} B_{n}$ in (4). $n$ is also the bus width, and

$$
n=m s+r
$$

in which $s$ is stride. $m$ equals $\lfloor n / s\rfloor . r$ means remainder, which equals $n \bmod s$.

The function $A(x)$ is defined as

$$
A(x)= \begin{cases}0 & x=0 \\ 1 & x>0\end{cases}
$$

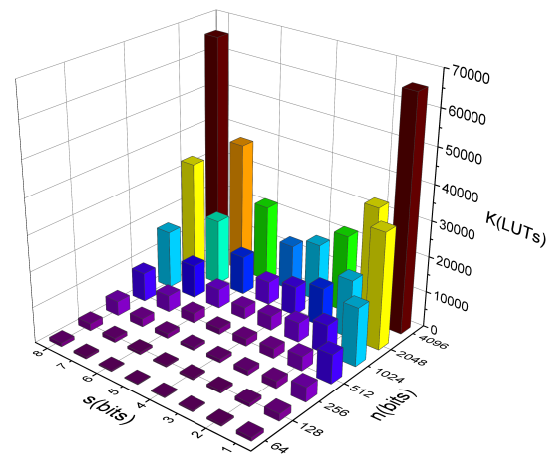

Fig. 4: The relationship between $K_{R_{1}}(n, s, 32), n$ and $s$.

and the resource utilization function $K(m, s, r, l)$ is defined as

$$
K(m, s, r, l)= \begin{cases}(l / 2) \cdot(m+A(r)) & s \leq 5 \\ (l / 2) \cdot\left(m \cdot 2^{s-5}+A(r)\right) & s>5, r \leq 5 \\ (l / 2) \cdot\left(m \cdot 2^{s-5}+A(r) \cdot 2^{r-5}\right) & s>5, r>5\end{cases}
$$

There are three equations corresponding to different $s$ and $r$. A single LUT is required to realize a logical table when $s$ is smaller than five, and cascaded LUTs are needed to realize a logical table when $s$ is larger than five. This is because a single LUT has five inputs. The computation of the remainder $r$ is the same as that of the stride $s . l$ is divided by 2 for the two outputs of a single LUT. (10) can be simplified as

$$
K(n, s, l)=\left\{\begin{array}{c}
(l / 2) \cdot(\lfloor n / s\rfloor+A(n \bmod s) \\
s \leq 5 \\
(l / 2) \cdot\left(\lfloor n / s\rfloor \cdot 2^{s-5}+A(n \bmod s)\right) \\
s>5, n \bmod s \leq 5 \\
(l / 2) \cdot\left(\lfloor n / s\rfloor \cdot 2^{s-5}+A(n \bmod s)\right. \\
\left.\cdot 2^{(n \bmod s)-5}\right) \quad s>5, n \bmod s>5
\end{array}\right.
$$

We should determine the values of $l$ and $s$ before exploring the relationship between $K$ and $s$ and find the best $s . l$ is related to the generator polynomial, and it is set to 32 here because CRC32 is the most widely used polynomial. The value of $n$ is set to $[64,128,256 \cdots 4096]$ to explore the influence of the bus width. The value of $s$ is set to $[1,2,3 \cdots 8]$. A stride larger than 8 will lead to an excessively large resource utilization. The resource utilization of Region 1 is $K_{R_{1}}(n, s, 32)$, and the relationship between $K_{R_{1}}(n, s, 32)$, $n$ and $s$ is illustrated in Fig. 4. As shown, stride-by-5 is optimal for any bus width. Stride-by-5 reduces the resource utilization by $79.69 \%-79.98 \%$ compared with stride-by-8, which is used in the slicing-by-4 and slicing-by- 8 algorithms[4].

The stride-by-5 algorithm is optimal for the 5-input LUTs in FPGA. Because the cascaded LUTs are needed if the stride is larger than 5, a single LUT cannot be fully used if the stride is smaller than 5. For the FPGAs with non-5-input LUTs (prior to Xilinx Virtex-5 or Altera Stratix-II), the stride defined by the number of LUT inputs should be used, and the LUT sharing mechanism should be exploited. The stride- 
by-5 algorithm is described in Algorithm 1; it processes the computation in Region 1 here, but the algorithm can also be used in Regions 3 and Region 4.

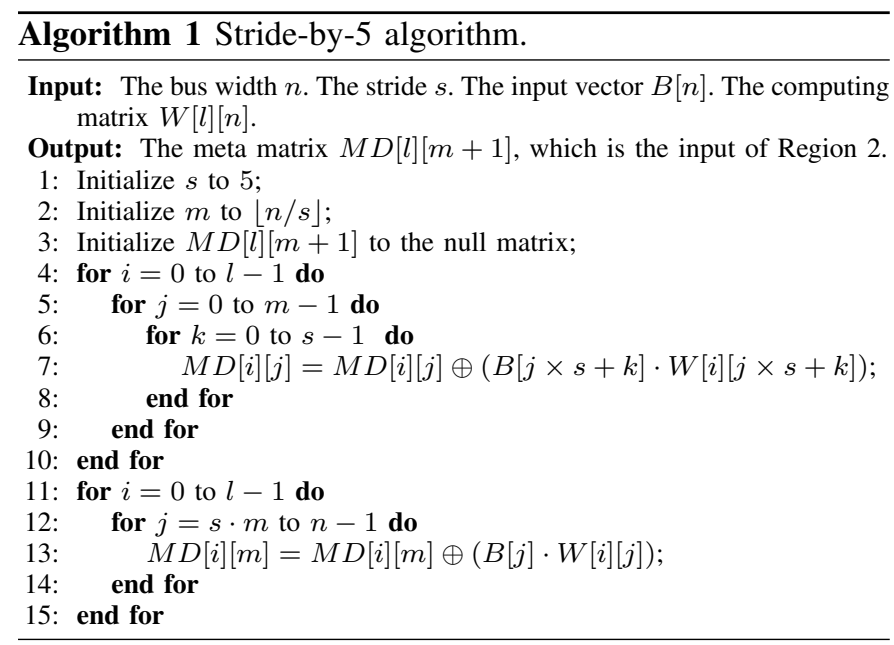

\section{Pipelining Go Back Algorithm}

In this section, the pipelining go back algorithm is proposed with an $O\left(\log _{2} n\right)$ resource utilization, and the derivation and description of the algorithm are presented.

The padding zeros problem is discussed in Section I. $p$ is used to represent the number of valid bits in the final word. $q$ is used to represent the number of padding zeros. The data vector of the final word is $B_{p+q}=\left[\mathrm{b}_{0}, \cdots, \mathrm{b}_{p-1}, 0, \cdots, 0\right]^{T}$. Substitute $B_{p+q}$ into (4), and then

$$
\begin{aligned}
C^{(p+q+k)} & =T^{p+q} C^{(k)}+W_{(p+q) n} B_{p+q} \\
& =T^{q}\left(T^{p} C^{(k)}+T^{p-1} S b_{0}+\cdots+S b_{p-1}\right) \\
& =T^{q} C^{(p+k)}
\end{aligned}
$$

The relationship between $C^{(p+k)}$ and $C^{(p+q+k)}$ is

$$
C^{(p+k)}=T^{-q} C^{(p+q+k)}
$$

There will be an $O(1)$ resource utilization to realize the computation of $T^{-q}$ because the size of $T^{-q}$ is $l \times l$ and has no relation with $n$. However, $q$ varies and $0 \leq q<n$, and if we use the $n$ table corresponding to every possible $q$, there will be an $O(n)$ resource utilization. We introduce a pipeline to reduce the resource utilization to $O\left(\log _{2} n\right)$.

Inspired by the binary representation, $q$ is represented as

$$
q=8 \cdot\left(x_{h-1} \cdot 2^{h-1}+x_{h-2} \cdot 2^{h-2}+\cdots+x_{1} \cdot 2+x_{0}\right)
$$

$q$ and $n$ are multiples of 8 because the data transfer in bytes. The value of $x$ can be 0 or 1 , and $h$ is the number of pipeline stages, which can be represented as

$$
h=\log _{2}(n / 8)
$$

(14) and (15) can be used to convert (13) to

$$
\begin{aligned}
C^{(p+k)} & =\left(\left(T^{-8 \cdot 2^{n-1}}\right)^{x_{n-1}} \cdots\left(T^{-8}\right)^{x_{0}}\right) C^{(p+q+k)} \\
& =\left(R_{1}^{x_{n-1}} \cdot R_{2}^{x_{n-1}} \cdots R_{h}^{x_{0}}\right) C^{(p+q+k)}
\end{aligned}
$$

where $\left[R_{1}, R_{2}, \ldots, R_{h}\right]$ is the $h$ matrices for the $h$-stage pipeline, and the size of each matrix is $l \times l$. $\left[R_{1}, R_{2}, \ldots, R_{h}\right]$ can be used to convert $C^{(p+q+k)}$ to $C^{(p+k)}$. Stride-by-5 algorithm can be used to convert $\left[R_{1}, R_{2}, \ldots, R_{h}\right]$ to the content of the LUTs. Using the resource utilization function in (11), the resource utilization of the pipeline is $K_{R_{4}}=$ $h \cdot K(l, s, l)$, where $R_{4}$ means the Region 4 in Fig. 2. $K_{R_{4}}$ can be represented as

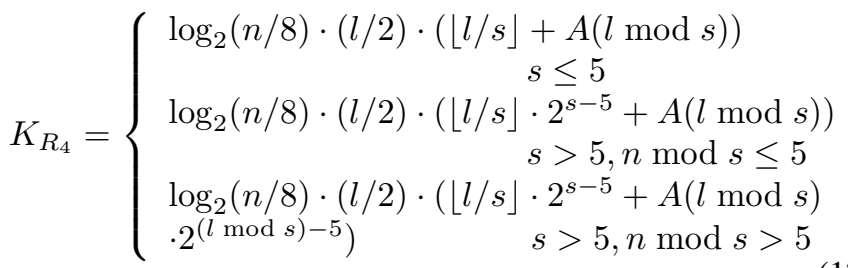

As shown in (17), we can achieve an $O\left(\log _{2} n\right)$ resource utilization using the pipelining go back algorithm. The algorithm is described in Algorithm 2.

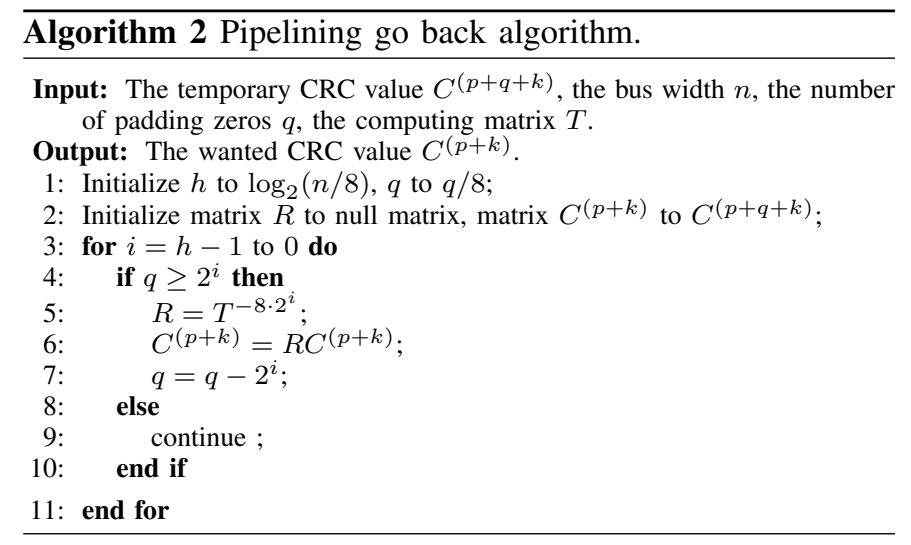

\section{Reprogramming by HWICAP}

Region 5 in Fig. 2 represents an HWICAP IP core, which can dynamically modify the content of the LUTs. It consumes 186 LUTs for any bus width. In contrast, configuration logic realized by logic resources leads to several thousands of LUTs being consumed when $n \geq 1024$ [5], and the resource utilization increases with increasing bus width. The operation procedure of reprogramming using the HWICAP IP core is described as follows:

1) Complete the initial design, generate the bitstream using Vivado, and download the bitstream into the FPGA;

2) Extract the locations of the LUTs used;

3) When reprogramming is needed, compute the new content of the LUTs using (4) and (16);

4) Map the content of the LUTs to the initial value of the LUTs;

5) Write the initial value to the LUTs using the AXI Lite interface of the HWICAP IP core.

The method of reprogramming by HWICAP is useful in engineering. Our contributions are as follows:

1) We verify the feasibility of reprogramming the FPGA implementation of the CRC algorithm using the HWICAP IP core. It leads to a small and constant resource utilization regardless of the bus width; 
2) The proposed method can change the CRC polynomial directly, without re-coding and re-synthesizing;

3) The code of the above procedure can be accessed in [1], as a part of the entire project. To our best knowledge, this is the first open source code covering the whole procedure described above.

\section{E. Segmented System Architecture}

Non-segmented system architecture can't process multiple frames in one word (clock), which decreases the throughput of short or misaligned frames. It is called bus efficiency problem. The segmented system architecture is proposed to solve the problem. The bus format is just like that in [7], and the block in [7] is another name for the segment in [18]. For an example, a 4096-bit bus can process 8 complete frames at the same time; hence, the bus can be divided into 8 regions[7]. The number of regions only depends on the bus width. Different segment widths are feasible, and if 64-bit segment width is chosen, one region can be divided into 8 segments (blocks). The proposed segmented system architecture is shown in Fig. 5. Compared with the proposed non-segmented system architecture, proposed segmented system architecture has slightly more complex Region 1 and Region 2, and multiple duplicates of Region 3 and Region 4. The number of duplicates is just the maximum number of the frames processed in a single word.

The comparison between the proposed segmented system architecture and the proposed non-segmented system architecture can be found in Fig. 6. The red cuboid represent the non-segmented system architecture. The blue cuboid represent the increment between the proposed segmented system architecture and the proposed non-segmented system architecture. The yellow slice (Bus width $=1024$, Segment width $=$ 512) represent the decrement between the two architectures. Fig. 6a shows that the increment in resource utilization mainly depends on the bus width instead of segment width. This is because the increment in resource utilization mainly depends the number of duplicates of Region 3 and Region 4, which only depends on the bus width. Fig. $6 \mathrm{~b}$ shows that the increment in 65-byte-frame throughput is obvious for most cases. The only decrese in throughput can be found when the bus width is 1024 bits and the segment width is 512 bits, where the two architectures have the same bus efficiency for 65-byte-frame throughput and the non-segmented architecture has a slightly higher frequency. 64 bits is chosen as the segment width in the rest of the brief. The dataset of the Fig. 6 can be found at [1].

\section{F. Packet Processing Flow of Two System Architecture}

In this section, the packet processing flow of the two system architectures are illustrated. The bus width of the two architectures is 4096 bits, and the segment width of the segmented system architecture is 64 bits (64 segments, 8 regions).

\section{Non-Segmented System Architecture}

The packet processing flow of non-segmented system architecture is described as follows:

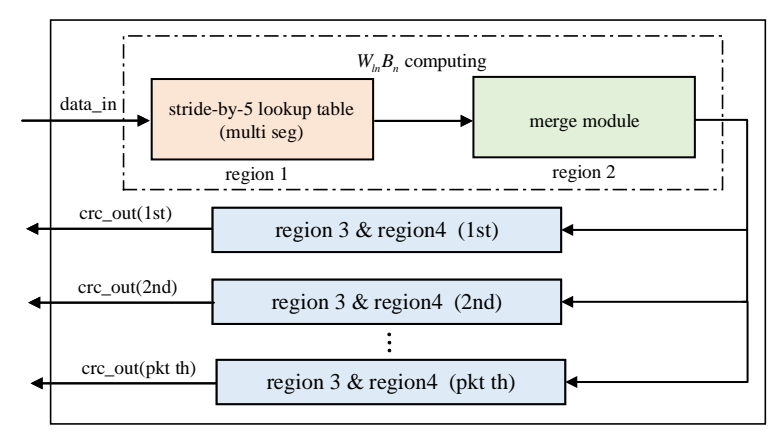

Fig. 5: Proposed segmented system architecture.

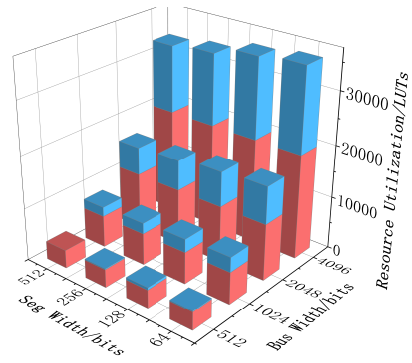

(a) Resource utilization.

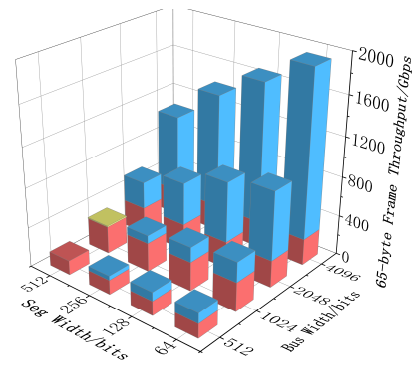

(b) 65-byte-frame throughput.
Fig. 6: Comparison between segmented architecture and nonsegmented architecture.

1) Region 1: Data input is composed of 512-bit frame and 3584-bit padding zeros; Region 1 compute the $M D[l][m+1]$ in Algorithm 1, where $l=32$ and $m=819$

2) Region 2: Data input is a $32 \times 820$ bit vector; Region 2 compute the $W_{l n} B_{n}$ in (4);

3) Region 3: Data input is the 32-bit $W_{l n} B_{n}$; Region 3 compute the $T^{n} C^{(k)}+W_{l n} B_{n}$ in (4);

4) Region 4: Data input is the 32-bit $T^{n} C^{(k)}+W_{l n} B_{n}$; Region 4 eliminates the impact of padding zeros ( (16) is used here ), and the correct CRC value is achieved.

\section{Segmented System Architecture}

The packet processing flow of segmented system architecture is described as follows:

1) Region 1: Data input is composed of 8 512-bit frames, which can be divided into 64 segments; Every segment has its own sub-region to compute the $M D[l][m+1]$ in Algorithm 1; Every $M D[l][m+1]$ is a $32 \times 13$ bit vector, and a small xor function is use to convert the $M D[l][m+1]$ to $W_{l n} B_{n}$.

2) Region 2: Data input is $64 W_{l n} B_{n}$ of 64 segments; Region 2 merge the $W_{l n} B_{n}$ of the same frame; $8 W_{l n} B_{n}$ of the 8 frames are achieved.

3) Region 3 and 4: Data input is the $8 W_{l n} B_{n}$ of 8 frames; Every $W_{l n} B_{n}$ has its own Region 3 and 4, and the Region 3 and 4 here do the same work as that of the non-segmented architectures. Finally, $8 \mathrm{CRC}$ values for 8 frames can be achieved. 


\section{EXPERIMENTAL RESULTS}

\section{A. Synthesis Result}

There are three state-of-the-art studies[7][5][20]. Architecture in [5][20] can be reprogrammed, whereas architecture in [7] cannot be reprogrammed. Two proposed architectures are implemented with Virtex-7 XC7VX690T, and [7][5][20] are implemented with Virtex-7 XCVH870T, Virtex6 XC6VLX550T and Stratix-V 5SGSED6N1F45I2, respectively. In this section, two proposed architecture are compared with their works in terms of resource utilization and maximum throughput. The proposed segmented architecture is compared with the architecture in [7] in terms of throughput on all frame lengths. Architecture in [7] and proposed two architectures are based on CRC32, and architecture in [5] is based on CRC64; hence, the resource utilization in [5] halves for a fair comparison. Power consumption of two proposed architectures is reported at last. In the following we use the $S A$ to refer to the segmented architecture.

The synthesis result is illustrated in Fig. 7. Fig. 7a shows that the resource utilization of the proposed non-SA is lower than that of architectures in [5] and [7] by $80.7 \%-87.5 \%$ and $25.1 \%-46.2 \%$, respectively. The proposed SA has a lower resource utilization by $81.7 \%-85.9 \%$ and $2.9 \%-20.8 \%$. The lower resource utilization results from the algorithms and method in Section 3, which can also guarantee high performance and programmability. If the programmability is not required, the resource utilization can be furtherly decreased. In contrast, the architecture in [5] is based on a slicingby-N algorithm whose stride is 8 , which will lead to a much higher resource utilization. Furthermore, the complex configuration logic of the architecture in [5] also leads to a considerable resource utilization. The architecture in [7] cannot be reprogrammed, which will reduce much resource utilization, even though its resource utilization is higher than that of two proposed architectures. Architecture in [7] is not concerned with the stride; hence, the LUT resource cannot be fully utilized, which leads to a higher resource utilization. Architecture in [20] has a lower resource utilization than that of the non-SA by $74.4 \%-81.3 \%$. The reasons for the lower resource utilization of [20] are: 1). [20] only need to process half filled and fully filled packets. In other word, the padding zeros problem is partly addressed. By contrast, two proposed architectures and [7][5] can fully address the padding zeros problem; 2). The cost of Nios II IP core is not taken into account in [20]. By contrast, proposed two architectures take the cost of HWICAP into account. Moreover, it is difficult to scale the bus width of [20] up to 1024 bits.

Fig. 7b shows that the maximum throughput of proposed non-SA is higher than that of the architecture in [5][7][20] by $24.2 \%-37.9 \%, 37.4 \%-75.0 \%$ and $259.4 \%-284.5 \%$, respectively. The maximum throughput of the proposed SA is higher than that of the architecture in [5][7] by $28.7 \%-30.2 \%$ and $32.2 \%-80.2 \%$, respectively. The higher frequency leads to higher throughput, and the proposed two architectures can achieve higher frequency for the well-arranged pipelines in Region 1, Region 2 and Region 4.
The throughput on frame lengths from 64 bytes to 256 bytes can be found in Fig. 7c. The architecture in [5] and proposed non-SA can't process multiple frames at the same time; hence, only the architecture in [7] and proposed SA are compared. The two architectures use 4096-bit bus width and 64-bit segment wdith, therefore they have the same bus efficiency. The proposed SA has a $80.2 \%$ higher frequency and throughput than the architecture in [7]. The lowest throughput of $1933.9 \mathrm{Gbps}$ is achieved when the frame length is 65 byte. The complete dataset with frame lengths from 64 bytes to 1518 bytes can be accessed at [1].

Power consumption of two proposed architectures is illustrated in Fig. 7d. The power consumption is composed of static power consumption and dynamic power consumption. The static power consumption varys from $0.32 \mathrm{~W}$ to $0.48 \mathrm{~W}$, and the dynamic power consumption increases linearly with the bus width. The power consumption of proposed SA has a faster growth than that of the proposed non-SA. This is because the resource consumption of the proposed SA increases faster than that of the proposed non-SA.

\section{B. Comparison with More Works}

[21] presents an implementation and analysis of encoder and checker of CRC8 which takes 16-bits of input data to create 24-bits code-word and can detect errors up to 8bits. [22] introduce a hardware architecture for parallel CRC computation. It can be configured for a different polynomial at any time externally. [23] introduces a design and development of parallel CRC algorithm for the hardware implementation on FPGA to meet the specifications for FC. The algorithm can process 128-bit parallel data in a block by broken it into four 32-bit data and calculate their CRC, respectively, based on the LFSR. The aim of [24] is to design and implement the CRC5 and CRC8 systems that are used for USB token packet and ATM protocols, respectively. A parallel pipelining method is used to implement the proposed CRC architecture for both CRC encoder and decoder systems to achieve high throughput data with optimized hardware resources.

These works are suitable for embedded systems for their low resource consumption. However, their performance is also limited. Moreover, the padding zeros problem can't be solved. The comparison between the four works and the two proposed architectures can be founded in Table I.

\section{Board-Level Implementation Result}

We have verified our design on the VC709 FPGA development board. The system topology is shown in Fig. 8 . The polynomial of Ethernet CRC32 is adopted. The bus width is 1024 bits. The clock frequency is $500 \mathrm{Mhz}$. The maximum throughput of the system is 512 Gbps. The final report generated by Vivado shows that the resource utilization of the CRC computing block in Fig. 8a is the same as the result in Fig. 7a. The reprogramming procedure will not be discussed in detail due to space limitations, and all the codes used here can be accessed at [1].

The correctness of the CRC computation and the system throughput should be verified. Sprient TestCenter N4U is used 
TABLE I: Comparison with more works

\begin{tabular}{|c|c|c|c|c|c|c|}
\hline & Bus width & Serial/Parallel & CRC polynomial & Frequency/Mhz & Throughput/Gbps & Resource/LUTs \\
\hline$[21]$ & 16 & parallel & CRC-8 & - & - & 13 \\
\hline$[22]$ & 16 & parallel & CRC-16 & 23.71 & 1.656 & 2771 \\
\hline$[23]$ & 128 & parallel+ serial & CRC-32 & - & - & 390 \\
\hline$[24]$ & $11 / 64$ & parallel & CRC-5/CRC-8 & 278.5 & $3.069 / 17.824$ & $96 / 801$ \\
\hline Proposed non-SA & $64-4096$ & parallel & CRC-32 & $508.0-576.7$ & $35.26-2080.77$ & $1074-19902$ \\
\hline Proposed SA & $512-4096$ & parallel & CRC-32 & $518.9-534.4$ & $273.64-2142.24$ & $3658-35912$ \\
\hline
\end{tabular}

TABLE II: Board-level test results

\begin{tabular}{|c|c|c|c|c|}
\hline Frame length /Bytes & 64 & 512 & 1518 & random \\
\hline L2 frames rate /Gbps & 7.62 & 9.62 & 9.86 & 9.75 \\
\hline Bus occupancy ratio (non-SA) & $2.98 \%$ & $1.88 \%$ & $1.94 \%$ & $2.05 \%$ \\
\hline Bus efficiency (non-SA) & $49.94 \%$ & $99.94 \%$ & $99.26 \%$ & $92.89 \%$ \\
\hline Throughput (non-SA) /Gbps & 255.7 & 511.7 & 508.2 & 475.6 \\
\hline Bus occupancy ratio (SA) & $1.49 \%$ & $1.88 \%$ & $1.93 \%$ & $1.91 \%$ \\
\hline Bus efficiency (SA) & $99.92 \%$ & $99.98 \%$ & $99.86 \%$ & $99.64 \%$ \\
\hline Throughput (SA) /Gbps & 511.6 & 511.9 & 511.3 & 510.1 \\
\hline Error frames checked & yes & yes & yes & yes \\
\hline
\end{tabular}

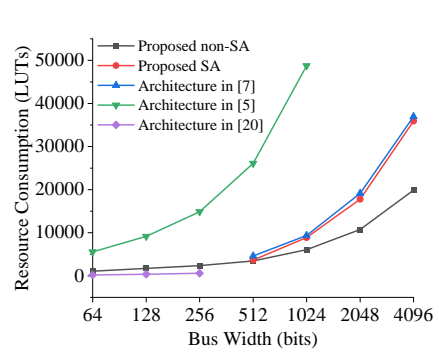

(a) Resource utilization.

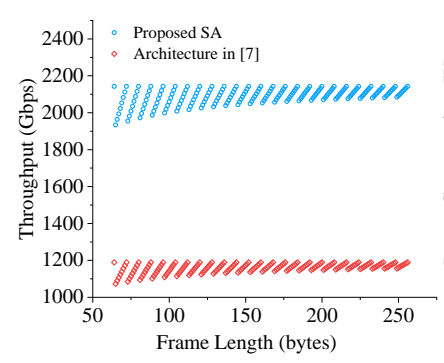

(c) Throughput and frame lengths.

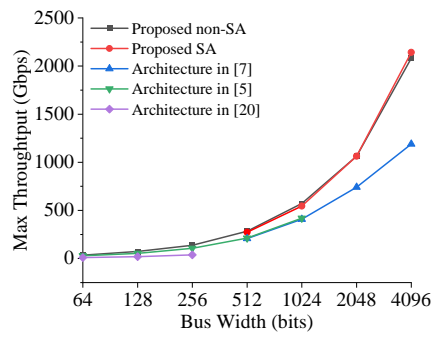

(b) Maximum throughput.

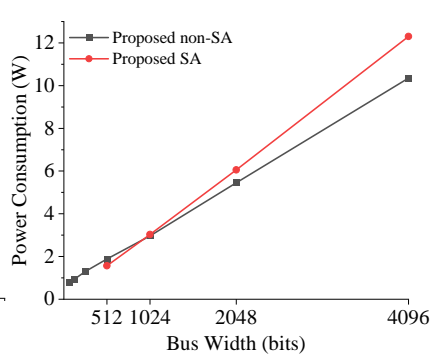

(d) Power consumption.

Fig. 7: Synthesis result.

to generate $10 \mathrm{Gbps}$ test frames with some error frames in them. The correctness can be verified if all the error frames can be checked out. The L2 frame rate is lower than $10 \mathrm{Gbps}$ (L1 frame rate) for the 20 byte frame gap. The statistics of the bus occupancy ratio are gathered. The L2 frame rate and bus occupancy ratio can be used to compute the bus efficiency, and the throughput can be inferred using the bus efficiency.

The test result is shown in Table II. It can be found that the SA can achieve a higher throughput than the non-SA, especially for the 64-byte frames. This is because the SA can achieve a higher bus efficiency. If the short or misaligned frames have a large share in the network traffic, SA should be adopted for a guaranteed throughput. Otherwise, non-SA is a better choice for its lower resource utilization.

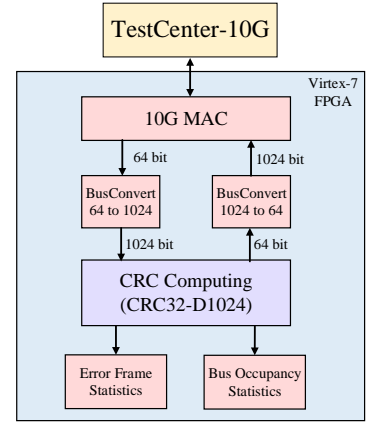

(a) System architecture.

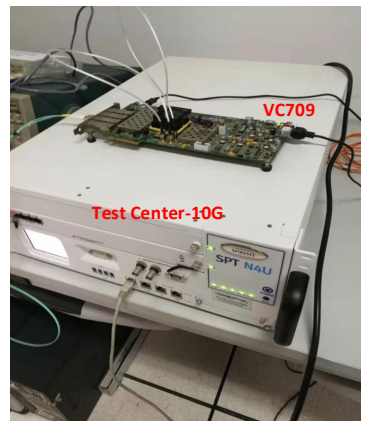

(b) VC709 and TestCenter.
Fig. 8: Board-level implementation.

\section{CONCLUSION AND FUTURE WORK}

Two algorithms and a method are proposed to realize lowcost, high performance, and programmable CRC computation. These algorithms and method can be used in segmented or non-segmented architectures. The synthesis results show that proposed architectures can achieve a lower resource utilization and higher throughput compared with two of the state-of-theart architectures. The board-level implementation results show that the theoretical low resource utilization, high throughput, and programmability can be achieved. The source code can be accessed at [1]. Our future work will focus on making the hardware reconfiguration method (HWICAP) technologyindependent.

\section{REFERENCES}

[1] S.C. of Low-Cost and Programmable CRC Implementation. Accessed: Oct. 24, 2019. [Online]. Available: https://github.com/FPGANetworking/Low-Cost-and-Programmable-CRC.

[2] D. Firestone, A. Putnam, S. Mundkur, D. Chiou, A. Dabagh, M. Andrewartha, H. Angepat, V. Bhanu, A. Caulfield, E. Chung et al., "Azure Accelerated Networking: SmartNICs in the Public Cloud," in 15th USENIX Symposium on Networked Systems Design and Implementation (NSDI 18), Apr. 2018, pp. 51-66.

[3] R. H. Dennard, F. H. Gaensslen, V. L. Rideout, E. Bassous, and A. R LeBlanc, "Design of ion-implanted MOSFET's with very small physical dimensions," IEEE J. Solid-State Circuits, vol. 9, no. 5, pp. 256-268, Oct. 1974 
[4] M. E. Kounavis and F. L. Berry, "Novel Table Lookup-Based Algorithms for High-Performance CRC Generation," IEEE Trans. Comput., vol. 57, no. 11 , pp. 1550-1560, Nov. 2008.

[5] A. Akagic and H. Amano, "High-speed fully-adaptable CRC accelerators," IEICE Trans. Inf.\& Syst., vol. 96, no. 6, pp. 1299-1308, 2013.

[6] T. Henriksson and D. Liu, "Implementation of fast CRC calculation," in Design Automation Conference, Jan. 2003.

[7] L. Kekely, J. Cabal, and J. Kořenek, "Effective FPGA Architecture for General CRC," in International Conference on Architecture of Computing Systems. Springer, 2019, pp. 211-223.

[8] J. Cabal, L. Kekely, and J. Kořenek, "High-Speed Computation of CRC Codes for FPGAs," in 2018 International Conference on FieldProgrammable Technology (FPT), Dec. 2018, pp. 234-237.

[9] C. Toal, K. McLaughlin, S. Sezer, and X. Yang, "Design and Implementation of a Field Programmable CRC Circuit Architecture," IEEE Trans. Very Large Scale Integr. (VLSI) Syst., vol. 17, no. 8, pp. 1142-1147, 2009.

[10] The P4 Language Consortium. (Nov. 2018). The P4 Language Specification, Version 1.0.5. [Online]. Available: https://p4.org/p4-spec/p414/v1.0.5/tex/p4.pdf.

[11] M. Grymel and S. B. Furber, "A Novel Programmable Parallel CRC Circuit," IEEE Trans. Very Large Scale Integr. (VLSI) Syst., vol. 19, no. 10, pp. 1898-1902, Oct. 2011.

[12] J. Jung, H. Yoo, Y. Lee, and I. Park, "Efficient Parallel Architecture for Linear Feedback Shift Registers," IEEE Trans. Circuits Syst. II, Exp. Briefs, vol. 62, no. 11, pp. 1068-1072, Nov. 2015.

[13] S. Gueron, "Speeding up CRC32C computations with Intel CRC32 instruction," Information Processing Letters, vol. 112, no. 5, pp. 179$185,2012$.

[14] P. Reviriego, A. Ullah, and S. Pontarelli, "PR-TCAM: Efficient TCAM Emulation on Xilinx FPGAs Using Partial Reconfiguration," IEEE Trans. Very Large Scale Integr. (VLSI) Syst., Aug. 2019.

[15] G. Campobello, G. Patane, and M. Russo, "Parallel CRC realization," IEEE Trans. Comput., vol. 52, no. 10, pp. 1312-1319, Oct. 2003.

[16] Zhenzhong Xiao, D. Koch, and M. Lujan, "A partial reconfiguration controller for altera stratix v fpgas," in 2016 26th International Conference on Field Programmable Logic and Applications (FPL), 2016, pp. $1-4$.

[17] K. Vipin and S. A. Fahmy, "FPGA Dynamic and Partial Reconfiguration: A Survey of Architectures Methods and Applications," ACM Comput. Surv., vol. 51, no. 4, pp. 1-39, 2018

[18] P. Orosz, T. Tóthfalusi, and P. Varga, "FPGA-Assisted DPI Systems: $100 \mathrm{Gbit} / \mathrm{s}$ and Beyond," IEEE Commun. Surveys Tuts., vol. 21, no. 2, pp. 2015-2040, 2019.

[19] Y. R. Qu and V. K. Prasanna, "High-Performance and Dynamically Updatable Packet Classification Engine on FPGA," IEEE Trans. Parallel Distrib. Syst., vol. 27, no. 1, pp. 197-209, Jan. 2015.

[20] M. Jubin and T. Nayak, "Reconfigurable very high throughput low latency VLSI (FPGA) design architecture of CRC 32," Integration, vol. 56, pp. 1-14, 2017.

[21] M. M. Arifin, M. T. Hasan, M. T. Islam, M. A. Hasan, and H. S. Mondal, "Design and implementation of high performance parallel crc architecture for advanced data communication," in 2019 4th International Conference on Electrical Information and Communication Technology (EICT), 2019, pp. 1-5.

[22] A. Sahu, R. S. Gamad, and P. P. Bansod, "Design and implementation of encoder and decoder for cyclic redundancy check," in 2019 International Conference on Communication and Electronics Systems (ICCES), 2019, pp. 2007-2011.

[23] W. Chuxiong and S. Haifeng, "Design and implementation of parallel crc algorithm for fibre channel on fpga," The Journal of Engineering, vol. 2019, no. 21, pp. 7827-7830, 2019.

[24] N. N. Qaqos, "Optimized fpga implementation of the crc using parallel pipelining architecture," in 2019 International Conference on Advanced Science and Engineering (ICOASE), 2019, pp. 46-51. 